\title{
Design and Simulation of the nuSTORM Pion Beamline
}

\author{
A. Liu $^{\mathrm{a}}$, D. Neuffer ${ }^{\mathrm{a}}$, A. Bross ${ }^{\mathrm{a}}$ \\ ${ }^{a}$ Fermilab, P.O.Box 500, Batavia, Illinois, 60510, USA
}

\begin{abstract}
The nuSTORM (neutrinos from STORed Muons) proposal presents a detailed design for a neutrino facility based on a muon storage ring, with muon decay in the production straight section of the ring providing well defined neutrino beams. The facility includes a primary high-energy proton beam line, a target station with pion production and collection, and a pion beamline for pion transportation and injection into a muon decay ring. The nuSTORM design uses "stochastic injection", in which pions are directed by a chicane, referred to as the Orbit Combination Section (OCS), into the production straight section of the storage ring. Pions that decay within that straight section provide muons within the circulating acceptance of the ring. The design enables injection without kickers or a separate pion decay transport line. The beam line that the pions traverse before being extracted from the decay ring is referred to as the pion beamline. This paper describes the design and simulation of the pion beamline, and includes full beam dynamics simulations of the system.
\end{abstract}

(C) 2015 Published by Elsevier Ltd.

Keywords: nuSTORM, pion beam line, muon storage ring, stochastic injection

\section{INTRODUCTION}

The goal of nuSTORM [1] is to provide well-defined neutrino beams for precise measurements of neutrino cross-sections and short-baseline (SBL) oscillations. A schematic drawing of the nuSTORM facility is presented in Figure 1. A high-intensity proton beam is directed onto a target, producing a large spectrum of secondary pions. Pions are focused by a collection lens and inserted through a chicane into the straight section of the storage ring. Pion decay within the straight section produces muons which are within the circulating acceptance of the storage ring, where they are stored for approximately the muon lifetime [2]. Muons that decay within the production straight section produce neutrino beams of known flux and flavor $\left(\mu^{+} \rightarrow e^{+}+\bar{v}_{\mu}+v_{e}\right.$ or $\left.\mu^{-} \rightarrow e^{-}+\bar{v}_{e}+v_{\mu}\right)$ that can be used for experiments at

\footnotetext{
*Corresponding author

Email address: aoliu@fnal.gov (A. Liu)
}

17 the near detector (ND in Figure 1) for neutrino cross8 section measurements and at the far detector (FD) site for SBL neutrino oscillation studies.

The critical design challenge is in storing the maximal number of muons. The nuSTORM solution is to design a large-acceptance storage ring (accepting $\delta P / P \approx \pm 10 \%)$ and to inject higher-momentum pions from a large-acceptance pion beamline (PBL) into the production straight section. The CERN, BNL and Fermilab g-2 experiments also use muons from pion decay in a ring $[3,4,5]$, however, the injection in the g2 experiments is different from that at nuSTORM. At nuSTORM, the injection is done through a beamline that is shared by pions with higher momenta $(\sim 5 \mathrm{GeV} / \mathrm{c})$ and muons with lower momenta $(\sim 3.8 \mathrm{GeV} / \mathrm{c})$. Pions are injected in a dispersive region of this shared beamline, and join with the muon orbits as their dispersion is suppressed to 0 . No kickers or septa are needed at the injection. All magnets used at nuSTORM are CW, while the g-2 experiments use pulsed magnets or fast kickers 


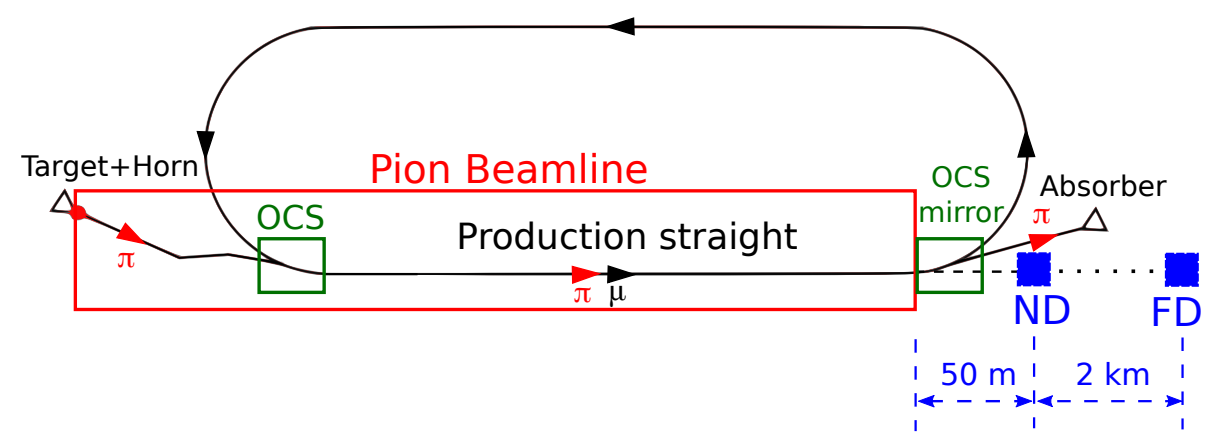

Figure 1 . The schematic of the beamline structure in the nuSTORM facility

to inject the beams. In addition, the shared beamline accepts a wide muon momentum band from pion decay, while the g-2 delivery rings accepts only a very limited momentum band of muons from forward decays.

The nuSTORM horn was optimized based on the design of this pion beamline, with consideration of the pion decay kinematics, to increase the number of useful muons at the end of the production straight [6]. Figure 2 shows the distribution of pions in the transverse phase space at the downstream face of the magnetic horn. An ellipse showing the fitted $2 \mathrm{~mm} \cdot \mathrm{rad}$ acceptance aperture of the muon decay ring is displayed.

Pions that decay into lower momentum muons within the ring acceptance provide the stored muons. In addition, the pion decay in the production straight provides a time-separated muon neutrino beam that is also available to the detectors. The subsequent background-free decays of the stored muons provide the electron and muon neutrino beams essential for precision neutrino studies. Positive or negative muons are stored by changing the polarity of the bending magnets, providing complementary neutrino sources. This injection through decay acceptance enables injection for multiple storage ring turns without kickers or capture rf, and muon accumulation can be reasonably large. This non-Liouvillean injection process was termed stochastic injection $[2,8]$.

\subsection{Discussion of the "Stochastic Injection" Concept}

Particle motion in accelerator physics is dominated by Hamiltonian dynamics within electromagnetic fields, which implies symplectic motion with conservation of phase space (Liouville theorem). Great improvements are possible when non-Liouvillean processes, such as

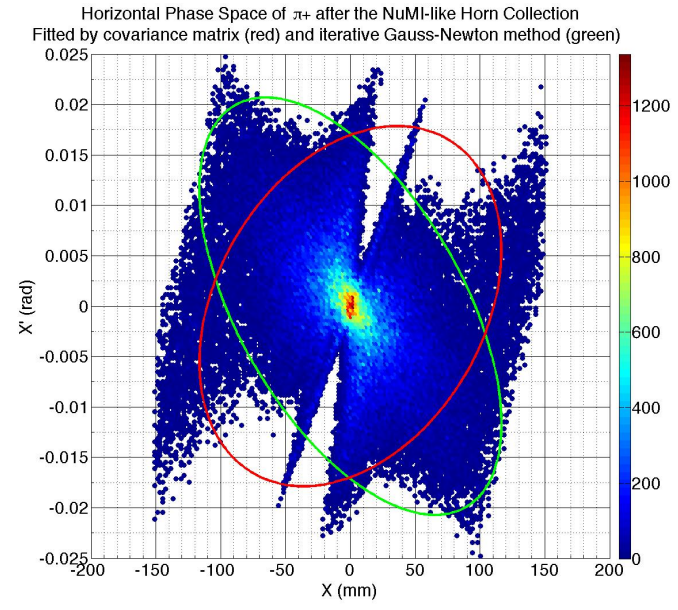

Figure 2. The distribution of $5 \pm 1 \mathrm{GeV} / \mathrm{c}$ pions in the transverse phase space, as generated using MARS15[7], and the corresponding fitted $2 \mu \mathrm{m} \cdot \mathrm{rad}$ Gaussian acceptance ellipse. The fitting results from the IGN method and the covariance matrix are shown in green and red, respectively. The IGN method conserves the core shape of the distribution.

beam cooling (by radiation damping, stochastic cooling and ionization or frictional cooling) and charge exchange injection, are enabled.

In $\mathrm{H}^{-}$charge exchange injection, stripping foil traversal transforms an $\mathrm{H}^{-}$ion into a proton $\left(\mathrm{H}^{+}\right)$of about the same energy, enabling multiturn accumulation of protons in the same initial phase space within a storage ring. The present concept is quite similar. An unstable particle inserted into a storage ring decays into 
a more stable one of different momentum. If the de- ${ }_{126}$ cayed momentum matches the momentum of the stored ${ }_{127}$ particles in the ring, the particle is added to the stored ${ }_{128}$ beam, increasing the stored beam phase-space density. ${ }_{129}$ The non-Liouvillean random process of particle decay ${ }_{130}$ enables multiturn stacking of particles within the same ${ }_{131}$ phase space. The name "stochastic injection" was introduced when this concept was first presented in 1980 $[2,8]$. The naming was influenced by the contemporary use of "stochastic" to describe other important nonLiouvillean beam handling techniques. (i.e., stochastic cooling) Although nuSTORM does not require multiturn pion injection, it can be used.

\section{PARAMETER SELECTION}

From studies of the neutrino beam requirements coupled with the detector sensitivity, the nuSTORM collaboration selected a muon storage ring center momentum of $3.8 \mathrm{GeV} / \mathrm{c}$. The ring is designed to have a large acceptance in $\delta P / P(10 \%)$ and in transverse phase space in order to maximize the flux of neutrinos. The choice of $3.8 \mathrm{GeV} / \mathrm{c}$ for the central momentum of the muons results in neutrino beams that cover much of the energy range of interest for cross section and SBL oscillation studies. A higher momentum, $5 \mathrm{GeV} / \mathrm{c}$ for example, would extend the neutrino beam energy coverage for cross-section measurements, but at the penalty of higher cost. $3.8 \mathrm{GeV} / \mathrm{c}$ is viewed as a good point in the costbenefit analysis. The PBL is designed to accept higher energy pions at a momentum $P_{\pi 0}$ matched to produce a maximal number of muons to be stored at the ring. It is also designed for a momentum spread of $\sim \pm 10 \%$ and a large transverse acceptance of $2000 \mu \mathrm{m} \cdot \mathrm{rad}$. Nevertheless, due to the stochastic feature of the injection scheme, even pions injected into the ring with as large as a $\pm 20 \%$ momentum spread have the possibility to decay to produce muons that are stored in the ring.

\subsection{Selection of the Pion Beam Central Momentum}

For an initial distribution, we assume that the pions are produced over the range $\left[0.9 P_{0}, 1.1 P_{0}\right]$ with a linear momentum probability density function of $f_{\mathbf{p}_{\pi}}\left(p_{\pi}\right)=$ $a p_{\pi}+b(a<0)$ (see Section 4). For illustration, the momentum distribution of pions at the downstream end of the nuSTORM baseline horn from MARS simulation is shown in Figure 3. A relativistic pion of momentum $p_{\pi}$ decays to a muon with the momentum of the muon randomly within the range $\left[p_{\min }\left(p_{\pi}\right), p_{\max }\left(p_{\pi}\right)\right]={ }_{138}$ $\left[0.573 p_{\pi}, p_{\pi}\right]$. The maximum and minimum momentum 139 of a pion that can decay to a muon with momentum 140 $p$ are $p_{\pi, \max } \approx 7 / 4 p, p_{\pi, \min } \approx p$. The difference $p_{\max }\left(p_{\pi}\right)-p_{\min }\left(p_{\pi}\right)$ can also be written as $2 p_{\mu}^{\prime} \gamma$, where $p_{\mu}^{\prime}=29.79 \mathrm{MeV} / \mathrm{c}$ is the momentum of the muon in the pion rest frame, and $\gamma=p_{\pi} / m_{\pi}^{\prime} \beta c$ is the Lorentz factor of the pion, $\beta \approx 1$. The momentum distribution of the muon is then $1 /\left(2 p_{\mu}^{\prime} \gamma\right)$ within that interval. The probability density function for obtaining muons of momentum $p$ from this pion beam is:

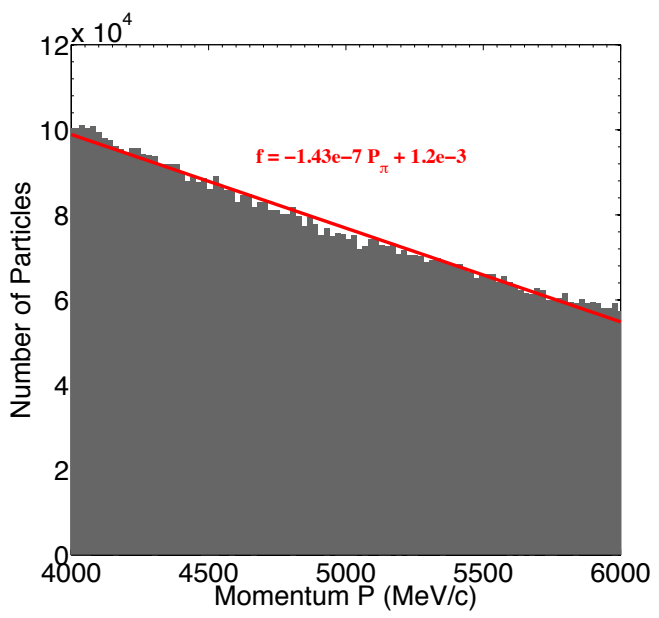

Figure 3. The momentum distribution of pions at the downstream end of the nuSTORM baseline horn and the fitted probability function as a function of pion momentum $p_{\pi}$.

$$
f_{\mathbf{p}}(p)=\int_{p_{1}}^{p_{2}}\left(a p_{\pi}+b\right) \cdot \frac{1}{2 \gamma p_{\mu}^{\prime}} d p_{\pi}
$$

where the limits of integration are determined by the step function limits in the pion beam and muon decay momenta. From these step function limits, we obtain a piecewise function for $f_{\mathbf{p}}(p)$ :

$$
f_{\mathbf{p}}(p)=\left\{\begin{array}{rl}
0 & p \leq 0.516 P_{0} \\
2.34 a\left[1.75 p-0.9 P_{0}\right]+2.34 b \ln \left(\frac{1.94 p}{P_{0}}\right) & 0.516 P_{0} \leq p \leq 0.63 P_{0} \\
2.34\left[0.2 P_{0} a+\ln (1.22) b\right] & 0.63 P_{0} \leq p \leq 0.9 P_{0} \\
2.34 a\left[1.1 P_{0}-p\right]+2.34 b \ln \left(\frac{1.1 P_{0}}{p}\right) & \\
0.9 P_{0} \leq p & \leq 1.1 P_{0} \\
0 & p \\
0 & \geq 1.1 P_{0}
\end{array}\right.
$$

The integrated muon density is greatest if the entire muon acceptance is within the central region $0.63 P_{0}<$ $p_{\mu}<0.9 P_{0}$, which occurs when $0.9 P_{0} \geq 4.18 \mathrm{GeV} / \mathrm{c}$ 
and $0.63 P_{0} \leq 3.42 \mathrm{GeV} / \mathrm{c}$, or $4.64 \leq P_{0} \leq 5.43 \mathrm{GeV} / \mathrm{c}$. 190 Since $a<0$, the preferred $P_{0}$ is biased toward smaller 191 values. However, larger $P_{0}$ reduces the required dis- 192 persion for separating the two orbits, and the transverse 193 divergence introduced by decay scales as $1 / P_{0}$. These ${ }_{194}$ criteria can be balanced at $P_{0}=\sim 5 \mathrm{GeV} / \mathrm{c}$, near the ${ }_{195}$ center of the band.

Using an estimate of $a=0$ and $b=1 / 0.2 P_{0}$ (uniform ${ }^{197}$ pion momentum distribution) and integrating $f\left(p_{\mu}\right)$ over 198 the $3.8 \times(1 \pm 0.1) \mathrm{GeV} / \mathrm{c}$ aperture within the central 199 band, we obtain an integrated acceptance of $\sim 0.36$ at 200 $P_{0}=5.0 \mathrm{GeV} / \mathrm{c}$. This is a useful first estimate for 201 the maximal possible muon acceptance per decayed 202 $5 \times(1 \pm 0.1) \mathrm{GeV} / \mathrm{c}$ pion within the injection straight. ${ }^{203}$ (Approximately $50 \%$ of the injected pions decay in the straight.)

\subsection{Decay straight lattice design}

The decay straight is designed as a FODO lattice 208 (with alternating gradient quadrupoles separated by drift 209 spaces). The FODO cells of the decay straight must 210 capture muons at $3.8 \mathrm{GeV} / \mathrm{c}$ from pion decay in the 211 straight, and confine the muon beam so that the muons 212 are efficiently stored in the ring for multiturn circulation 213 before decay. The FODO lattice is designed to accept 214 both $3.8 \mathrm{GeV} / \mathrm{c}$ muons and $5.0 \mathrm{GeV} / \mathrm{c}$ pions. Therefore, 215 two sets of periodic Twiss parameters are needed for the 216 FODO structure. The straight section is simultaneously 217 matched with the rest of the decay ring at $3.8 \mathrm{GeV} / \mathrm{c}$ and ${ }_{218}$ to the pion beam line at $5.0 \mathrm{GeV} / \mathrm{c}$. [9]

The maximum decay angle of muons with respect 220 to the pion direction is approximately $7.9 \mathrm{mrad}$ at this 221 energy. This suggests that if we want to capture the 222 muons, we should design the acceptance angle for the 223 muon beam to be at least 7.9 mrad. This implies 224 that $\sqrt{\epsilon_{a c c} \gamma_{x, y}}=\sqrt{\epsilon_{a c c}\left(1+\alpha_{x, y}^{2}\right) / \beta_{x, y}} \geq 0.0079$, where $\alpha_{x, y}, \beta_{x, y}, \gamma_{x, y}$ are the Twiss parameters, and $\epsilon_{a c c}$ is the acceptance of the beamline. This criterion is satisfied for $\epsilon_{a c c}=0.002$ if $\beta_{x, y} \leq 32 \mathrm{~m}$.

On the other hand, it is desirable to measure the angular divergence of the muon beam to an accuracy of $0.1 \%$ of the neutrino production angle, which is inversely 230 proportional to the muon momentum in the lab frame. For muons with momentum $3.8 \mathrm{GeV} / \mathrm{c}$, the decay angle is approximated by $1 / \gamma_{\mu}=0.028 \mathrm{rad}$; thus the accuracy ${ }^{232}$ goal is $\delta_{x^{\prime}}<\sim 2.8 \times 10^{-5}$. The divergence of the ${ }^{233}$ beam is determined from measurement of the emittance ${ }^{234}$ of the beam, and we estimate that the accuracy of the ${ }_{235}$ measurement of the emittance is limited to $\delta \epsilon / \epsilon>\sim 1 \%$. ${ }_{236}$ With $x^{\prime}$ proportional to $\epsilon_{r m s}^{1 / 2}$, then $\delta x^{\prime} / x^{\prime} \sim 0.5 \%$. We ${ }_{237}$ require that $\sqrt{\epsilon_{r m s} \gamma_{x, y}} \leq 0.0056$. With $\epsilon_{r m s}=\epsilon_{a c c} / 6$ this translates into a requirement of $\beta_{\min }>10.6 \mathrm{~m}$.

A complementary requirement is that the beam divergence from the beam emittance be much smaller than the intrinsic decay divergence $\left(\sim 1 / \gamma_{\mu}\right)$, for neutrinos from muon decay. This requirement can be written as $\sqrt{\epsilon_{r m s} \gamma_{x, y}}<<0.028$. It can be satisfied by choosing $\beta_{x, y}>20 \mathrm{~m}$, where the rms beam divergence is less than $\sim 0.004$. The $4 \mathrm{mrad}$ beam divergence, in quadrature with the $28 \mathrm{mrad}$ intrinsic decay divergence, has only a small effect on the number of neutrinos reaching the detectors. It also allows us to determine the neutrino flux with less than $1 \%$ uncertainty by accurately measuring the muon beam emittance.

\subsection{Selection of FODO Straight Lattice Parameters}

The Decay Straight is composed of a FODO lattice ( $\mathrm{F}$ and $\mathrm{D}$ quads interspersed with $\mathrm{O}$ drifts) with equal $x$ and $y$ focusing. The lattice is determined by selecting $\beta_{\max }\left(\beta_{x}\right.$ at the $\mathrm{F}$ quad) and $\beta_{\min }\left(\beta_{x}\right.$ at the $\mathrm{D}$ quad), following the constraints discussed above. A thorough search within the possible solution space was done using MADX[10], to establish lattice parameters, and G4Beamline[11] to track beam properties. Beam propagation along a $150 \mathrm{~m}$ long straight was simulated and the properties of the muon beam in the production straight were recorded, for periodic Twiss parameters with different $\beta_{\min }$ and $\beta_{\max }$. The criteria used to determine the production straight parameters are summarized in Table 1

Solutions with $\beta_{\min }<20 \mathrm{~m}$ were disfavored. A range of acceptable solutions within $24 \mathrm{~m}<\beta_{\max }<32$ $\mathrm{m}$ and $20 \mathrm{~m}<\beta_{\min }<28 \mathrm{~m}$ was found. A broad optimum was found around $\beta_{\max } \sim 30 \mathrm{~m}, \beta_{\min } \sim 23 \mathrm{~m}$ and those values were used in the storage ring design. These values correspond to a FODO lattice with a cell length of $7 \mathrm{~m}$ and a phase advance per cell of $\sim 15^{\circ}$. For the same FODO cell, the corresponding periodic Twiss parameters for $5 \mathrm{GeV} / \mathrm{c}$ can then be found. The matched lattice and the Twiss parameters for the two reference momenta are shown in Figure 4.

\section{PION INJECTION BEAMLINE AND MUON STORAGE RING}

\subsection{Injection Beamline Optics Design}

From the pion optics of the FODO cells, we design the pion injection matching section. This is done by matching the optics backwards from the straight section to the pion collection horn. A large dispersion value $D_{x}$ is needed to separate the reference orbits of the 

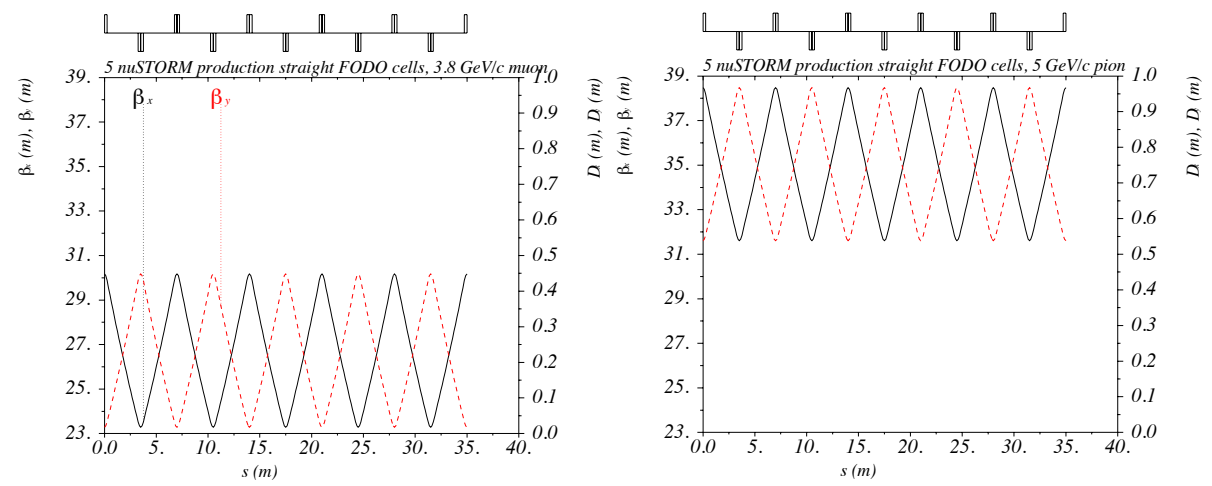

Figure 4. Twiss parameters $\beta_{x}, \beta_{y}$ in the nuSTORM production straight section, showing 5 complete FODO cells (35m) with $\beta_{x}, \beta_{y}$ for $3.8 \mathrm{GeV} / \mathrm{c}$ muons shown on the left, and for $5.0 \mathrm{GeV} / \mathrm{c}$ pions on the right.

\begin{tabular}{ll}
\multicolumn{2}{c}{ Table 1. Criteria for determining the parameters of the production straight straight periodic Twiss functions } \\
\hline Goal & Action \\
\hline Number of muons within the momentum acceptance after first pass & Maximize \\
Neutrino intensity at the detectors (Angular divergence of the muon beam) & Maximize (Minimize) \\
Number of magnets needed & Minimize \\
\hline
\end{tabular}

pion and muon beams. We require an orbit separation 266 of $\sim 0.4 \mathrm{~m}$ at the beam pipe split, which corresponds 267 to $D_{x} \sim 2.5 \mathrm{~m}$ from the tracking results. This $D_{x}{ }_{268}$ is generated by the combination of a bending dipole 269 and a horizontally defocusing quadrupole. A horizontal focusing quadrupole at the end of the injection beamline is included to match the beam into the FODO straight section. These quadrupoles and dipoles, with the drifts between them, are termed the Orbit Combination Section (OCS).

With the large $D_{x}$ generated in the OCS, the Twiss parameters $\beta_{x}, \beta_{y}$ must be minimized to control the total beam size. The Twiss parameters and $D_{x}$, matched back from the decay straight through the OCS for the reference $3.8 \mathrm{GeV} / \mathrm{c}$ muons, are displayed in Figure 5. Schematic views of the pion and muon orbits through the OCS are displayed in Figure 6. The sector dipole fields and edge angles for both muon and pion orbits are separately determined for matching muons into the ring and pions into the PBL, respectively. The off-axis passage of the pion beam through the quad is treated as a gradient dipole.

The pion beamline from the collection horn to the ${ }^{286}$ production straight is shown in Figure 7. The first dipole ${ }_{287}$ removes primary protons and wrong-sign pions from the 288 collected beam. The three dipoles are matched to form 289 an achromat (zero-dispersion). The quads are used to 290 control the beam size of the large-emittance pion beam 291 and for horn to straight optical matching. The Twiss parameters from the magnetic horn through the OCS and into the decay straight are displayed in Figure 8 for a $5.0 \mathrm{GeV} / \mathrm{c}$ reference pion.

\section{SIMULATION RESULTS AND DISCUSSION}

The MARS15 simulation code was used to simulate pion production in the target and generate the pion distributions at the downstream end of the production horn. We have used G4Beamline for simulation of particle transport and decay through the PBL, obtaining the muon distributions that can be captured in the storage ring. G4Beamline provides a relatively complete and realistic model for magnetic fields and apertures as well as particle decay and interactions. In the MARS simulation [4], a $39 \mathrm{~cm}$ long cylindrical Inconel target (about two interaction lengths, with a radius of $3 \mathrm{~mm}$ ) was bombarded with $2.4 \times 10^{7} 120 \mathrm{GeV}$ protons. The center of the target was shifted $3 \mathrm{~cm}$ toward the upstream side of a NuMI-like, $300 \mathrm{~cm}$ long magnetic horn. The number of pions in the momentum range of $5 \pm 1 \mathrm{GeV} / \mathrm{c}$ is 0.29 per POT. The pions and the muons from their decay are tracked in the PBL.

At the end of the decay straight FODO cells, a total of 0.05 muons per POT were obtained. With the transverse phase space and momentum acceptance cuts, 0.013 muons per POT were found within both $2 \mathrm{~mm} \cdot \mathrm{rad}$, 



Figure 5. Twiss parameters $D_{x}, \beta_{x}, \beta_{y}$ (left: $3.8 \mathrm{GeV} / \mathrm{c}$ muon optics, right: $5.0 \mathrm{GeV} / \mathrm{c}$ pion optics) matched backwards from the first production straight FODO cell to the beginning of the OCS.

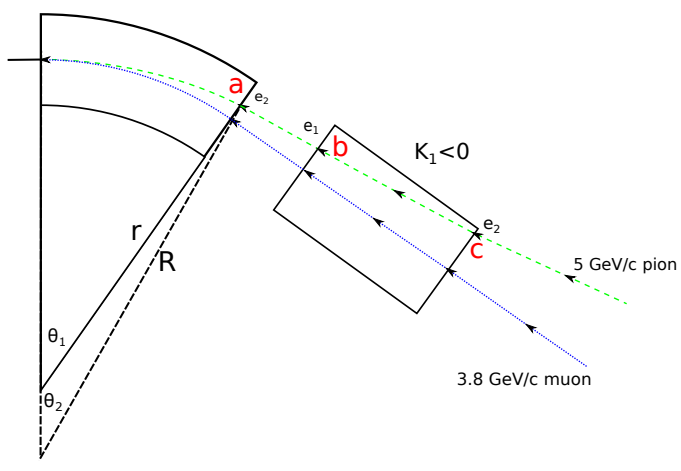

Figure 6. View of reference orbits in the OCS magnets. The injected pions or circulating muons move from right to left, following the arrows.

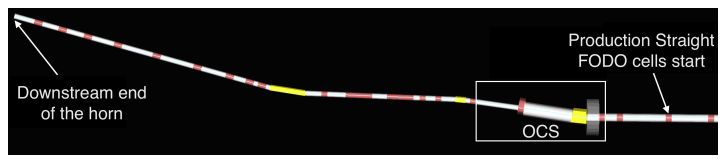

Figure 7. The layout of the Pion Beamline elements from the magnetic horn (leftmost) through the OCS quads and dipole (right). The white, yellow and red blocks represent drift tubes, dipoles, and quadrupoles, respectively. G4Beamline was used to generate the figure.

and $3.8 \times(1 \pm 0.1) \mathrm{GeV} / \mathrm{c}$. Plots of the muon distribution 30 in X-Y and X-X' space, and the histogram of the total 302 momentum distribution are shown in Figure 9. The ${ }_{303}$ muons within the momentum range of $5 \times(1 \pm 0.1) \mathrm{GeV} / \mathrm{c} 304$ are extracted, with the remaining pions, at the end of 305

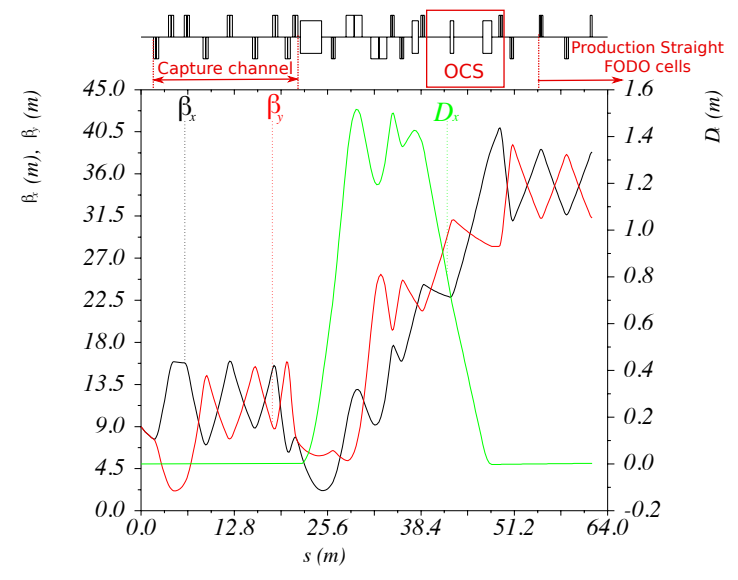

Figure 8. The $5.0 \mathrm{GeV} / \mathrm{c}$ pion optics from the collection horn to the focusing quad in the production straight FODO cell. Pions move from left to right. The last five magnets are within the ring.

decay straight along by a mirror image of the OCS, which also matches into the return arc. These pions and muons can be used in external beam experiments. After a degrader, the remaining lower energy muons can possibly be used in a future muon cooling experiment (see Figure 9). The neutrino fluxes at a 100 Tons Near Detector (ND) are plotted in Figure 10. nuSTORM can produce many orders of magnitudes more $v_{e}$ events than collected to date [12]. 



Figure 9. The X-Y real space (upper left), X-X' phase space (upper right) and momentum (lower) distributions of muons at the end of the production straight. The data corresponds to $2.4 \times 10^{7} 120 \mathrm{GeV}$ POT. The green momentum band $(3.8 \mathrm{GeV} / \mathrm{c}$ with a $\pm 10 \%$ acceptance bite) is the momentum acceptance range of the decay ring, and the red band $(5 \mathrm{GeV} / \mathrm{c}$ with a $\pm 10 \%$ acceptance bite) is the OCS mirror extraction momentum range.

\subsection{Variations and other applications}

The nuSTORM injection and storage ring is adapted to produce $\sim 2 \mathrm{GeV}$ neutrinos, but the concept can be readily changed to obtain neutrinos from $\sim 100 \mathrm{MeV}$ to tens of $\mathrm{GeV}$ with modifications of the injection beam and storage ring parameters. It is desirable that the muons be somewhat relativistic to minimize the decay ${ }_{324}$ emittance dilution.

The stochastic injection method could also be used to 325 accumulate ions in a storage ring by inserting unstable ${ }_{326}$ radioactive ions into a storage ring, which decay to a ${ }_{327}$ more stable state by beta-decay, $\left(X_{1} \rightarrow X_{2}+e^{-(+)}+v\right)$. ${ }_{328}$ The ions would be separable at the ends of the straight ${ }_{329}$

\section{ACKNOWLEDGMENTS}

We are grateful to the nuSTORM collaboration for their support of this research. We thank S.Y. Lee, M. Geelhoed, E. Gianfelice-Wendt, S. Striganov, P. Snopok and A. Bogacz for their discussions about the design. Fermilab is operated by Fermi Research Alliance, LLC 


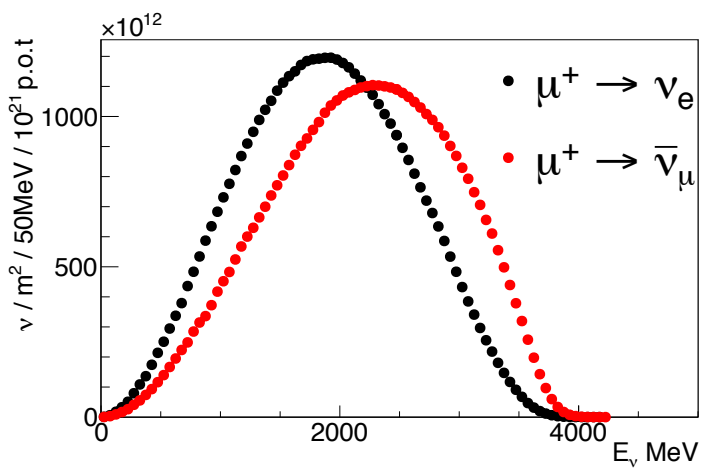

[13] E. Wildner, et al., Design of a neutrino source based on betabeams, Phys. Rev. ST Accel. Beams 17.

Figure 10. Neutrino flux from $\mu^{+}$decay at the near detector (50 meters downstream the end of the production straight).

under Contract No. DE-AC02-07CH11359 with the U. S. Department of Energy. This research was also partly funded by research grants DE-FG02-12ER41800 and NSF Phys 1205431.

\section{References}

[1] D. Adey, et al., nustorm-neutrinos from stored muons, Proposal to the Fermilab Physics Advisory committeeArXiv:1308.6822.

[2] D. Neuffer, Design considerations for a muon storage ring, Proc. Neutrino Mass Miniconference (V. Barger and D. Cline, ed.) Telemark, WI (1980) 199.

[3] F. J. Farley, E. Picasso, The muon (g-2) experiments, Annua Review of Nuclear and Particle Science 29 (1) (1979) 243-282.

[4] J. Grange, et al., Muon (g-2) Technical Design ReportarXiv:1501.06858.

[5] J. Grange, The New Muon g-2 Experiment at FermilabarXiv:1501.03040.

[6] A. Liu, A. Bross, D. Neuffer, Optimization of the magnetic horn for the nustorm non-conventional neutrino beam using the genetic algorithm, submitted to Nuclear Instruments and Methods in Physics Research A.

[7] N. Mokhov, et al., Mars15 overview, FermilabConf-07/008-AD, AIP Conf. Proc. 50 (2007) 896, http://www.ap.fnal.gov/MARS/.

[8] D. Neuffer, Design of muon storage rings for neutrino oscillations experiments, IEEE Trans. NS-28 (1981) 2034

[9] A. Liu, D. Neuffer, A. Bross, S. Lee, Determining the pion reference momentum for nustorm injection design, FERMILABTM-2563-APC (unpublished) (2013).

[10] F. Schmidt, H. Grote, MAD-X and Upgrade from MAD8, Proc. US Part. Acc. Conf., Portland, Ore, USAHttp://mad.home.cern.ch/mad/.

[11] T. Roberts, G4beamline - A "Swiss Army Knife" for Geant4, optimized for simulating beamlines, http://www.muonsinternal.com/muons3/G4beamline.

[12] D. Adey, R. Bayes, A. Bross, P. Snopok, nustorm and a path to a muon collider, Annual Review of Nuclear and Particle Science 65 (1) (2015) null. arXiv:http://dx.doi.org/10.1146/annurevnucl-102014-021930, doi:10.1146/annurev-nucl-102014 021930.

URL http://dx.doi.org/10.1146/annurev-nucl-102014-021930 